\title{
INFLUÊNCIA DE PLANTAS FORRAGEIRAS NA PRODUÇÃO DE MASSA DE MUDAS DE Albizia lebbeck (L.) Benth
}

Marcos Vinícius Miranda Aguilar - aguilarmarcos2009@hotmail.com Instituto Federal de Educação, Ciência e Tecnologia do Norte de Minas Gerais IFNMG, Campus Salinas

Marília Dutra Massad - mariliamassad@ yahoo.com.br Instituto Federal de Educação, Ciência e Tecnologia do Norte de Minas Gerais IFNMG, Campus Salinas

Tiago Reis Dutra - tiagoreisdutra@ gmail.com Instituto Federal de Educação, Ciência e Tecnologia do Norte de Minas Gerais IFNMG, Campus Salinas

Pedro Henrique Pereira - pedro_taiobeiras@yahoo.com.br Instituto Federal de Educação, Ciência e Tecnologia do Norte de Minas Gerais IFNMG, Campus Salinas

Eduarda Soares Menezes - eduarda_menezs@ hotmail.com Universidade Federal dos Vales do Jequitinhonha e Mucuri - UFVJM

Aline Ramalho dos Santos - alineramalho13@ hotmail.com Universidade Federal do Espírito Santo - UFES 


\section{RESUMO}

O presente trabalho teve como objetivo avaliar o efeito de diferentes graus de competição com as espécies Brachiaria brizantha e Brachiaria decumbens no crescimento inicial da albízia. Adotou-se o delineamento experimental de blocos ao acaso, sendo sete tratamentos e cinco repetições, totalizando 35 parcelas ou vasos. Os tratamentos foram: T1 ausência de matocompetição; T2 - baixa ocorrência de Brachiaria brizantha; T3 - média ocorrência de Brachiaria brizantha; T4 - alta ocorrência de Brachiaria brizantha; T5 - baixa ocorrência de Brachiaria decumbens; T6 - média ocorrência de Brachiaria decumbens; e T7 - alta ocorrência de Brachiaria decumbens. Aos 90 dias de convivência foi quantificada a produção de massa seca da parte aérea, raiz e total das espécies forrageiras e das mudas de albízia. As mudas de albízia, em plantio isolado apresentaram maiores produções de massa seca da parte aérea, raiz e total. A presença da Brachiaria brizantha e Brachiaria decumbens afetou o desenvolvimento da espécie florestal. As maiores produções de massa seca da parte aérea nas forrageiras foram observadas em alta e média ocorrência de Bachiaria brizantha e Brachiaria decumbens.

Palavras-chave: Albizia lebbeck (L.) Benth, Áreas degradadas, Brachiaria brizantha, Brachiaria decumbens, Plantas daninhas.

\section{INTRODUÇÃO/OBJETIVO}

A degradação do solo pode estar relacionada com práticas agrícolas intensivas que provocaram grave degradação ambiental, incluindo perda da biodiversidade e poluição de mananciais superficiais e subterrâneos, devido ao uso intenso de fertilizantes e pesticidas nas lavouras (FAO, 2011). O desconhecimento de práticas conservacionistas, a ausência de planejamento a médio e longo prazo, a maior pressão pelo uso do solo, dentre outros fatores, favorecem com que as áreas que são usadas nos cultivos agrícolas e pecuária sejam abandonadas, permitindo o estabelecimento de plantas espontâneas (SCHUMACHER et al., 2005).

A recuperação das áreas degradadas pode ser conceituada como um arranjo de açõesidealizadas e executadas por diversos especialistas, que visam proporcionar o restabelecimento das condições de equilíbrio, e sustentabilidade presentes anteriormente em 


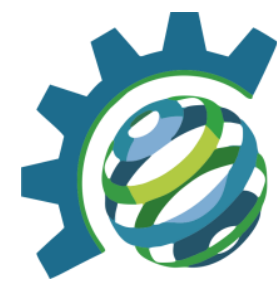

um determinado sistema natural (ATTANASIO et al., 2006). Para escolher as espécies que serão utilizadas nessas áreas deve-se levar em consideração os estudos da composição florística da vegetação remanescente da região.

Dentre as espécies com alto potencial para a recuperação de áreas degradadas destaca.se a Albizia lebbeck (L.) Benth, popularmente conhecida como albízia pertence à família Fabaceae e sub família Mimosoideae. Caracteriza-se por apresentar um rápido crescimento, habilidade para fixar nitrogênio e melhorar a estrutura do solo, tendo usos múltiplos e facilidade para consórcio com culturas agrícolas (DUTRA et al., 2008). Essa espécie possui alto potencial em sistemas silvipastoris, principalmente por apresentar forragem de alta qualidade; promover sombra, que beneficia diretamente os animais, reduzindo a temperatura no verão e estimulando o crescimento das pastagens (PAULINO et al., 2008). É utilizada na arborização urbana em estacionamentos, praças e margens de rodovias. Além de ser boa produtora de néctar para a produção de mel em apiários, sua rusticidade aliada ao crescimento rápido a tornam muito procurada para cultivo (NASCIMENTO, 2009). Essa espécie florestal, como qualquer outra população natural, está sujeita a uma série de fatores ecológicos que podem afetar o seu desenvolvimento e a obtenção dos produtos desejados, seja de modo direto ou indireto. Dentre esses fatores podemos citar $\mathrm{pH}$ do solo, luminosidade e competição entre as espécies

Muitas das áreas em que se realiza a restauração florestal encontram-se ocupadas com elevada massa de gramíneas exóticas invasoras que dificultam o estabelecimento das plantas nativas, e em geral, precisam ser contundentemente controladas, pois sua presença é a principal causa de insucesso das iniciativas de restauração florestal (CORNIH \& BURGIN, 2005). Em grande parte das áreas florestais, as populações das plantas infestantes atingem elevadas densidades populacionais e passam a condicionar fatores que são negativos ao crescimento e produtividade das árvores e à operacionalização do sistema produtivo (MARCHI et al., 2002).

Em projetos de recuperação áreas de degradadas principalmente em pastagens, há necessidade de pesquisas para desenvolver novas técnicas a serem utilizadas para o controle dos competidores, com o propósito de reduzir ao máximo os impactos ambientais com a relação de mato-competição e a efetividade do plantio, empregando técnicas capazes de controlar as plantas invasoras, e simultaneamente acelerar o desenvolvimento das mudas. 


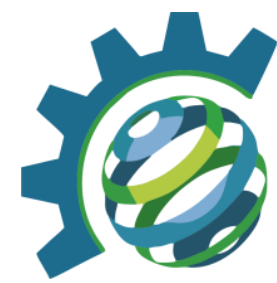

Diante do exposto, o presente trabalho teve como objetivo avaliar o efeito de diferentes graus de competição com as espécies Brachiaria brizantha e Brachiaria decumbens no crescimento inicial de albízia.

\section{METODOLOGIA}

O experimento foi instalado no "Viveiro de Produção de Mudas Florestais" do Instituto Federal de Educação, Ciência e Tecnologia do Norte de Minas Gerais (IFNMG), Campus Salinas. Adotou-se o delineamento experimental de blocos ao acaso, sendo sete tratamentos e cinco repetições, totalizando 35 parcelas ou vasos. Os tratamentos variaram de acordo com o número de sementes de Brachiaria brizantha e Brachiaria decumbens, sendo: T1 - ausência de matocompetição; T2 - baixa ocorrência de matocompetição com Brachiaria brizantha; T3 - média ocorrência de matocompetição com Brachiaria brizantha; T4 - alta ocorrência de matocompetição com Brachiaria brizantha; T5 - baixa ocorrência de matocompetição com Brachiaria decumbens; T6 - média ocorrência de matocompetição com Brachiaria decumbens; e T7 - alta ocorrência de matocompetição com Brachiaria decumbens.

As mudas de albízia com idade de 9 meses foram transplantadas em vasos com capacidade de 18 litros, preenchidos previamente com solo. Na mesma ocasião, foram plantadas a $2 \mathrm{~cm}$ de profundidade as sementes de Brachiaria brizantha e Brachiaria decumbens. Para as condições de baixa, média e alta ocorrência de matocompetição, foi considerada a presença de três, seis e nove plantas invasoras por vaso, respectivamente.

Foi realizada uma adubação dois dias antes do transplantio das mudas para os vasos aplicando-se, por solução, $200 \mathrm{mg} \mathrm{dm}^{-3}$ de $\mathrm{P}, 100 \mathrm{mg} \mathrm{dm}^{-3}$ de $\mathrm{K}$ e $40 \mathrm{mg} \mathrm{dm}^{-3}$ de S, tendo como fontes $\mathrm{NaH}_{2} \mathrm{PO}_{4} \cdot \mathrm{H}_{2} \mathrm{O}$ (fosfato de sódio), $\mathrm{KH} 2 \mathrm{PO} 4$ (potássio hidrogenofosfato) e $\mathrm{K}_{2} \mathrm{SO}_{4}$ (sulfato de potássio), respectivamente, conforme sugerido por Passos (1994). Posteriormente, aplicou-se também uma solução de micronutrientes nas seguintes doses: $0,81 \mathrm{mg} \mathrm{dm}^{-3} \mathrm{de} B$ $\left(\mathrm{H}_{3} \mathrm{BO}_{3}\right.$; ácido bórico), 1,33 $\mathrm{mg} \mathrm{dm}^{-3} \mathrm{de} \mathrm{Cu}\left(\mathrm{CuSO}_{4} .5 \mathrm{H}_{2} 0\right.$; sulfato de cobre $), 0,15 \mathrm{mg} \mathrm{dm}^{-3} \mathrm{de}$ Mo [ $\left.\left(\mathrm{NH}_{4}\right)_{6} \mathrm{Mo}_{7} \mathrm{O}_{24} \cdot 4 \mathrm{H}_{2} \mathrm{O}\right)$; molibdato de amônio], 3,66 mg dm${ }^{-3}$ de $\mathrm{Mn}\left(\mathrm{MnCl}_{2} \cdot \mathrm{H}_{2} \mathrm{O}\right.$; cloreto de manganês) e 4,0 mg dm${ }^{-3}$ de $\mathrm{Zn}\left(\mathrm{ZnSO}_{4} \cdot 7 \mathrm{H}_{2} 0\right.$; sulfato de zinco), de acordo com Alvarez et al. (2006). 


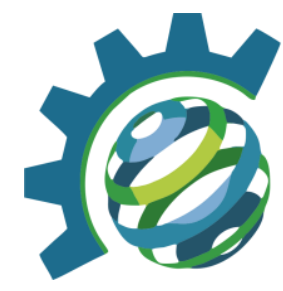

Aos 90 dias de convivência, as espécies forrageiras e as mudas de albízia foram retiradas dos vasos, lavadas em água corrente e secas em estufa de circulação forçada de ar, a aproximadamente $65^{\circ} \mathrm{C}$, até peso constante para quantificação da massa seca da parte aérea (MSPA), massa seca da raiz (MSR) e massa seca total (MST).

Os dados foram submetidos à análise de variância e, quando o efeito foi significativo, as médias foram comparadas pelo teste de Tukey $(\mathrm{p}<0,05)$. As análises estatísticas foram realizadas utilizando-se o pacote ExpDes.pt (FERREIRA et al., 2013) do software livre R (R CORE TEAM, 2015).

\section{RESULTADOS E DISCUSSÃO}

As variáveis massa seca da parte aérea (MSPA), massa seca da raiz (MSR) e massa seca total (MST) de mudas de albízia observou-se diferenças significativas entre os tratamentos estudados (Tabela 1).

Tabela 1 - Produção de massa seca da parte aérea (MSPA), massa seca da raiz (MSR) e massa seca total (MST) de mudas de albízia em plantio isolado ou em competição com Brachiaria brizantha e Brachiaria decumbens aos 90 dias

\begin{tabular}{lccc}
\hline \multirow{2}{*}{ Tratamentos $^{2}$} & MSPA $^{1}$ & MSR & MST \\
\cline { 2 - 4 } & \multicolumn{3}{c}{ g planta $^{-1}$} \\
\hline Plantio isolado & $42,56 \mathrm{a}$ & $36,82 \mathrm{a}$ & $79,37 \mathrm{a}$ \\
BO- Brachiaria brizantha & $20,11 \mathrm{~b}$ & $23,42 \mathrm{~b}$ & $43,53 \mathrm{~b}$ \\
MO- Brachiaria brizantha & $14,62 \mathrm{~b}$ & $20,07 \mathrm{bc}$ & $34,69 \mathrm{~b}$ \\
AO- Brachiaria brizantha & $12,18 \mathrm{~b}$ & $17,81 \mathrm{bc}$ & $29,98 \mathrm{~b}$ \\
BO- Brachiaria decumbens & $17,28 \mathrm{~b}$ & $21,60 \mathrm{~b}$ & $38,88 \mathrm{~b}$ \\
MO- Brachiaria decumbens & $13,99 \mathrm{~b}$ & $16,96 \mathrm{bc}$ & $30,94 \mathrm{~b}$ \\
AO-Brachiaria decumbens & $11,33 \mathrm{~b}$ & $12,31 \mathrm{c}$ & $23,64 \mathrm{~b}$ \\
\hline
\end{tabular}

${ }^{1}$ Médias seguidas da mesma letra na coluna não diferem entre si pelo Teste Tukey a $5 \%$ de probabilidade. ${ }^{2}$ Plantio isolado de albízia; baixa (BO), média (MO) e alta (AO) ocorrência de Brachiaria brizantha; baixa (BO), média (MO) e alta (AO) ocorrência de Brachiaria decumbens

A albízia em plantio isolado apresentou maior produção de MSPA, MSR e MST se diferenciando significativamente dos demais tratamentos (Tabela 1). Esses resultados mostram que a convivência das espécies Brachiaria brizantha e Brachiaria decumbens promoveram prejuízo ao desenvolvimento inicial da albízia. Fagioli et al. (2000), constataram que a Brachiaria decumbens e $B$. brizantha apresentaram um efeito inibitório no incremento de massa seca da raiz de guandu (Cajanus cajan). Em estudos com Brachiaria decumbens, 
Moreira \& Bragança (2011) evidenciaram que a mesma torna-se planta indesejável, quando utilizada nas áreas que não sejam de pastagens, em função de sua rusticidade e do difícil controle.

Com o aumento da densidade das plantas forrageiras observou-se diminuição no incremento em MSR para as mudas de albízia. A menor média para a variável MSR foi encontrada em alta ocorrência de Brachiaria decumbens, entretanto, não se diferenciando estatisticamente dos tratamentos de alta e média ocorrência de Brachiaria brizantha e média ocorrência de Brachiaria decumbens. Segundo Carvalho et al. (2014), a habilidade competitiva das plantas é determinada pela espécie, ou a sua densidade de ocorrência nas áreas cultivadas. A redução na acumulação de MST está relacionada com a menor produção de MSPA, e MSR da espécie florestal em convivência com essas forrageiras. Gustafson et al. (2004) afirmam que a elevada velocidade de emergência, e de crescimento inicial das plantas forrageiras as tornam prioritárias na utilização dos recursos do meio e, por isso, geralmente elas levam vantagem na utilização destes.

As espécies forrageiras no plantio em competição com mudas de albízia apresentaram diferença significativa na produção de MSPA (Tabela 2).

Tabela 2 - Massa seca da parte aérea (MSPA) de Brachiaria brizantha e Brachiaria decumbens em plantio em competição com mudas de albízia aos 90 dias

\begin{tabular}{lc}
\hline \multicolumn{1}{c}{ Tratamentos $^{2}$} & MSPA $^{1}$ \\
\cline { 2 - 2 } & g planta $^{-1}$ \\
\hline BO- Brachiaria brizantha & $74,23 \mathrm{~b}$ \\
MO- Brachiaria brizantha & $81,41 \mathrm{ab}$ \\
AO-Brachiaria brizantha & $91,99 \mathrm{ab}$ \\
BO- Brachiaria decumbens & $75,40 \mathrm{~b}$ \\
MO-Brachiaria decumbens & $89,50 \mathrm{ab}$ \\
AO- Brachiaria decumbens & $93,60 \mathrm{a}$ \\
\hline
\end{tabular}

${ }^{1}$ Médias seguidas da mesma letra na coluna não diferem entre si pelo Teste Tukey a $5 \%$ de probabilidade. ${ }^{2}$ Baixa (BO), média (MO) e alta (AO) ocorrência de Brachiaria brizantha; baixa (BO), média (MO) e alta (AO) ocorrência de Brachiaria decumbens

As maiores médias nas plantas forrageiras em competição com as mudas de albízia para a variável MSPA foram encontradas no tratamento de alta ocorrência de Brachiaria decumbens, mas não se diferenciou estaticamente dos tratamentos de alta e média ocorrência de Bachiaria brizantha e média ocorrência de Brachiaria decumbens (Tabela 2). Matos et al., 


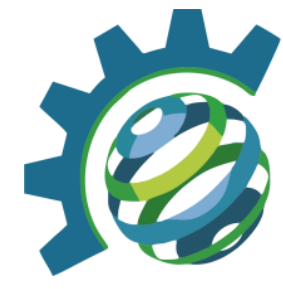

(2013) evidenciaram que a espécie Brachiaria decumbens em competição com Coffea arabica apresentou maior habilidade competitiva quando em maior densidade, reduzindo a taxa fotossintética, a condutância estomática e a transpiração do cafeeiro. Com o aumento da densidade de plantas de Brachiaria brizantha convivendo com a espécie florestal constatou-se redução nos valores observados para essas variáveis (CRUZ, 2014). Além disso, as espécies do gênero Brachiaria são perenes, agressivas, resistentes e por isso, são consideradas importantes espécies competidoras. A Brachiaria em maior densidade apresenta alto potencial de interferência com as mudas de albízia. Isto pode ser explicado pelo fato da Brachiaria possuir características morfológicas como hábito de crescimento ereto, maior altura e taxa de crescimento que as mudas de albízia, fato que a favorece na competição por luz.

Os menores incrementos em MSPA nas forrageiras foram encontrados nos tratamentos de baixa ocorrência de Brachiaria brizantha e Brachiaria decumbens, em consorciação com as mudas de albízia (Tabela 2).

\section{CONCLUSÕES/RECOMENDAÇÕES}

As mudas de albízia, em plantio isolado apresentaram maiores produções de massa seca da parte aérea, raiz e total. A presença da Brachiaria brizantha e Brachiaria decumbens afetou o desenvolvimento da espécie florestal.

As maiores produções de massa seca da parte aérea nas forrageiras foram observadas em alta e média ocorrência de Bachiaria brizantha e Brachiaria decumbens.

\section{REFERÊNCIAS BIBLIOGRÁFICAS}

ALVAREZ, V.H.; DIAS, L.E.; LEITO, P.B.; SOUZA, R.B.; RIBEIRO JUNIOR, E.S. Poda de raízes e adubação para crescimento do cafeeiro cultivado em colunas de solo. Revista Brasileira de Ciência do Solo, v.30, n.1, p.111-119, 2006.

ATTANASIO, C.M.; RODRIGUES, R.R.; GANDOLFI, S.; NAVE, A.V. Adequação Ambiental De Propriedades Rurais Recuperação de Áreas Degradadas Restauração de Matas Ciliares. Universidade De São Paulo. Departamento De Ciências Biológicas Laboratório De Ecologia E Restauração Florestal. Piracicaba, Julho 2006. Disponível em: < 


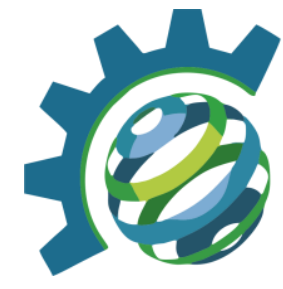

http://www.esalq.usp.br/gerd/Recuperacao/ApostilaTecnicoLERFFinal1.pdf> Acesso em Maio de 2017.

CARVALHO, L.B.; BIANCO, S.; BIANCO, M.S. Estudo comparativo do acúmulo de massa seca e macronutrientes por plantas de Zea mays e Ipomoea hederifolia. Planta Daninha, v.32, n.1, p. 99-107, 2014.

CORNISH, P.S.; BURGIN, S. Residual effects of glifosato herbicide in ecological restoration, Restorantion Ecology, Malden, v. 13, n. 4, p. 695-702, 2005.

CRUZ, L.R. Aspectos competitivos em espécies florestais: interferência de plantas daninhas e de brotações indesejadas. Dissertação (Mestrado em Produção Vegetal, concentração em Produção Vegetal) - Instituto de Ciências Agrárias da Universidade Federal de Minas. Montes Claros, MG, p. 40-43, 2014.

DUTRA, A.S.; FILHO, S.M.; DINIZ, F.O. Germinação de sementes de Albízia (Albízia lebbeck (L.) Benth) em função da luz e do regime de temperatura. Revista Caatinga, v.21, n.1, p.75-81, 2008.

FAGIOLI, M.; RODRIGUES, T.J.D.; ALMEIDA, A.R.P.; ALVES, P.L. Efeito inibitório da Brachiaria decumbens Stapf. Prain. e B. brizantha (Hochst ex a. Rich.) Stapf. cv. marandu sobre a germinação e vigor de sementes de guandu (Cajanus cajan (L.) Millsp.). Boletim da Indústria Animal, Nova Odessa, v.57, n.2, p.129-137, 2000.

FAO - FOOD AND AGRICULTURE ORGANIZATION OF THE UNITED NATIONS. 2011. The state of the world's land and water resources for food and agriculture. Roma: FAO. 50p.

FERREIRA, E.B.; CAVALCANTI, P.P.; NOGUEIRA, D.A. ExpDes.pt: experimental designs package $\mathrm{R}$ package version (1.1.2). 2013. 


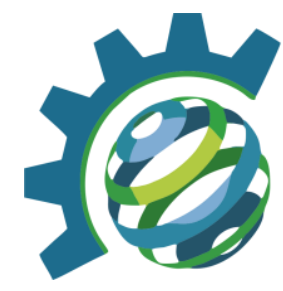

GUSTAFSON, D.J.; GIBSON, D.J.; NICKRENT, D.L. Competitive relationships of Andropogon gerardii (Big Bluestem) from remnant and restored native populations and select cultivated varieties. Functional Ecology, v. 18, n. 3, p. 451-457, 2004.

MARCHI, S.R.; PITELLI, R.A.; BEZUTTE, A.J.; CORRADINE, L.; ALVARENGA, S.F. Efeito de períodos de convivência e de controle das plantas daninhas na cultura de Eucalyptus grandis. In: SEMINÁRIO SOBRE CULTIVO MÍNIMO DO SOLO EM FLORESTAS, 1., 2002, Curitiba. Anais... Curitiba: EMBRAPA-CNPF, 2002. p. 122-133.

MATOS, C.C.; FIALHO, C.M.T.; FERREIRA, E.A.; SILVA, D.V.; SILVA, A.A.; SANTOS, J.B.; FRANÇA, A.C.; GALON, L. Physiological characteristics of coffee plants in competition with weeds. Bioscience Journal, v. 29, n. 5, p. 1111-1119, 2013.

MOREIRA, H.J. da C.; BRAGANÇA, H.B.N. Manual de Identificação de Plantas Infestantes. Cultivos de Verão. Campinas - SP: FMC, 2011. 1017p.

NASCIMENTO, L.S. Ecologia de Bruchidae na predação pré-dispersão de sementes de Albizia lebbeck (Benth.) em arborização. Seropédica, Rio de Janeiro, 2009. 73 f. Dissertação (Mestrado em Ciências) - Universidade Federal Rural do Rio de Janeiro, Seropédica, Rio de Janeiro, 2009.

PASSOS, M.A.A. Efeito da calagem e de fósforo no crescimento inicial da algaroba (Prosopis juliflora (SW) DC). 1994. 57f. Tese (Doutorado em Fitotecnia) - Universidade Federal de Viçosa, Viçosa, MG, 1994.

PAULINO, V.T.; BRAGA, G.J.; LUCENA, M.A.C.; GERDES, L.; COLOZZA, M.T. Sustentabilidade de pastagens consorciadas - ênfase em leguminosas forrageiras. In: PAULINO, V.T.; BRAGA, G.J.; LUCENA, M.A.C. et al., (Org.). II Encontro Sobre Leguminosas Forrageiras. 1 ed. Nova Odessa: IZ/APTA/SAA, 2008, v.1, p.1-55. 


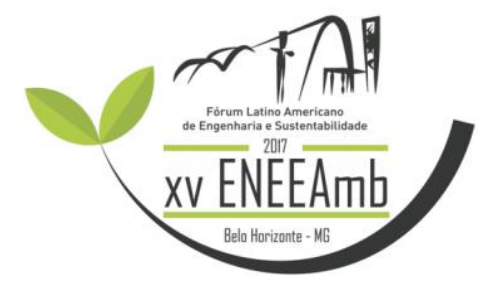

R CORE TEAM. R: A language and environment for statistical computing. Vienna: $R$ Foundation for Statistical Computing, 2015. Disponível em: http://www.R-project.org/ Acesso em 15 maio 2017.

SCHUMACHER, M.V. CALIL, F.N. VOGEL, H. L. M. Universidade Federal de Santa Maria Centro de Ciências Rurais. Departamento de Ciências Florestais. Silvicultura Aplicada. Santa Maria, maio de 2005. Disponível em: < $\underline{\text { http://www.celso- }}$ foelkel.com.br/artigos/outros/13_UFSM.\%20apostila_Silvicultura_aplicada.pdf> Acesso em Maio de 2017. 